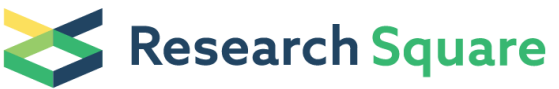 \\ Preprints are preliminary reports that have not undergone peer review. \\ They should not be considered conclusive, used to inform clinical practice, or referenced by the media as validated information.
}

\section{Characteristics of Clostridioides difficile infection in inflammatory bowel disease}

\section{Chenjie Tang}

Wuxi Children's Hospital

\section{Chengcheng Liu}

Jiangsu Province Hospital and Nanjing Medical University First Affiliated Hospital

\section{Yaping Han}

Jiangsu Province Hospital and Nanjing Medical University First Affiliated Hospital

Xiaohui Zhang

Jiangsu Province Hospital and Nanjing Medical University First Affiliated Hospital

\section{Wenying Xia}

Jiangsu Province Hospital and Nanjing Medical University First Affiliated Hospital

\section{Fang Ni}

Jiangsu Province Hospital and Nanjing Medical University First Affiliated Hospital

Genyan Liu ( $\boldsymbol{\sim}$ liugenyan@njmu.edu.cn )

Jiangsu Province Hospital and Nanjing Medical University First Affiliated Hospital https://orcid.org/0000-0002-8711-1322

\section{Research}

Keywords: Molecular epidemiology, risk factors, inflammatory bowel disease, Clostridioides difficile infection

Posted Date: June 30th, 2020

DOI: https://doi.org/10.21203/rs.3.rs-38076/v1

License: (1) (1) This work is licensed under a Creative Commons Attribution 4.0 International License.

Read Full License 


\section{Abstract}

Background: The epidemiology of Clostridioides difficile infection(CDI) in China is different from western countries and the characteristics of CDI among inflammatory bowel disease (IBD) in China may be unique. The aim of this study was to investigate the molecular epidemiology and to find out the risk factors of CDI among IBD inpatients in Jiangsu Province, China.

Methods: Patients with IBD admitted to the First Affiliated Hospital with Nanjing Medical University from August 2013 to December 2018 were enrolled. IBD patients were matched with non IBD patients according to age and gender. Diarrhea samples were sent for CDI diagnosis and the molecular epidemiology investigation was performed. Finally, patients' information was collected and logistic regression analysis was performed to analyze the independent risk factors of CDI in IBD patients.

Results: In this study, the incidence of CDI in IBD patients was much higher than that in non IBD patients (24.6\% vs. 9.0\%) and community acquired infection was the main kind. The predominant type of epidemic strain of $C$. difficile in this study was ST54. The shorter history of IBD and recent use of quinolone antibiotics were independent risk factors for CDI among diarrhea patients with IBD.

Conclusion: If the duration of IBD is within one year or quinolone antibiotics have been used recently, clinicians should consider the possibility of IBD patients complicated with CDI and adjust the treatment plan.

\section{Background}

Clostridioides difficile (C. difficile) is a kind of gram-positive spore-forming anaerobe bacillus. In 1978, $C$. difficile was found to be associated with pseudomememous enteritis and antibiotic-associated diarrhea[1]. The symptoms of Clostridioides difficile infection (CDI) include asymptomatic carriers, diarrhea, toxic megacolon and even death. In America, the status of $C$. difficile has exceeded methicillinresistant Staphylococcus aureus (MRSA) and has become the major cause of nosocomial infection[2, 3]. In mainland China, the incidence of CDI in diarrhea patients is about 14\%[4]. Moreover, the hypervirulent strain (NAP1/RT027) has been scatterly reported in China[5, 6]. On the other hand, the incidence of inflammatory bowel disease (IBD) in China has been increasing recently and it has become a common cause of digestive system disease including chronic diarrhea. More and more studies have shown that intestinal microecological imbalance, such as the decrease of beneficial bacteria and their metabolites in the intestinal tract and the increase of harmful bacteria and their metabolites, may change the intestinal environment, thus causing IBD. And this process is very similar to the occurrence and development of CDI[7]. However, it is not clear whether there is a causal relationship between CDI and IBD. Most studies have reported that IBD is an independent risk factor for CDI even in the absence of other factors, such as antibiotics and long-term hospitalization[8-10]. Additionally, more and more studies found an increasing incidence of CDI among patients with IBD and this kind of parients commonly had a more severe course of disease than non IBD population [9, 11-13]. 
In western countries, $\mathrm{CDI}$ is considered to be the main cause of hospital-acquired infections and the detection of CDI is widely performed. However, in China, due to the high nutritional requirements, anaerobic culture and the high cost of toxin detection, only some teaching hospitals carry out the project of $\mathrm{CDI}$ and physicians usually ignore this issue. Compared with western countries, such as Europe and the United States, China has its own social characteristics, such as aging population and extensive use of antibiotics, which are the rik factors of CDI. So, the molecular epidemiology of CDI among diarrhea patients with IBD in China may be unique. However, the incidence and risk factors of CDI among Chinese IBD patients are still poorly characterized. Therefore, we performed this study to investigate the molecular epidemiology and risk factors of CDI among hospitalized diarrhea patients with IBD in Jiangsu Province, China.

\section{Methods}

\section{Patients}

Adult patients with IBD hospitalized in the First Affiliated Hospital with Nanjing Medical University between August 2013 and December 2018 were enrolled in the current study. The inclusion criteria for IBD patients were as follows: (1) hospitalized IBD patients with ulcerative colitis (UC) or Crohn's disease (CD) within the study period; (2) at least 16 years of old; (3) with an increase of at least three loose and watery stools per day. To compare the incidence of CDI between IBD inpatients and non IBD inpatients, non IBD inpatients were mateched with IBD inpatients one by one according to gender and age (within 1 year). Patients with normal feces and incomplete information were excluded. This study was approved by the hospital ethics committee.

\section{Detection of CDI}

A case of CDI was defined on the basis of the presence of symptoms (usually diarrhea) and successful isolation of $C$. difficile with a positive PCR test for toxin genes. Toxigenic culture was performed as the standard diagnostic test for $\mathrm{CDI}[14,15]$. Isolation and toxin genes detection of $\boldsymbol{C}$. difficile were perfprmed as we reported before[16]. Briefly, $C$. difficile was isolated from all loose or watery fecal samples with cefoxitin-cycloserine fructose agar plates (BioMérieux, France) under anaerobic conditions. Suspected clones were further identified by VITEK 2 ANC cards (BioMérieux, France). Bacterial genomic DNA was extracted from the identified colonies using the TIANamp Bacteria DNA Kit (TIANGEN Biotech, Beijing, China) according to the manufacturer's instructions. Then, $t c d A$, $t c d B$, and binary toxin ( $c d t A / c d t B)$ genes were amplified and analyzed as previously described[17]. ATCC BAA-1870 was positive control and ATCC 700057 was negative control. All isolated strains and extracted necleic acid were frozen at $-70^{\circ} \mathrm{C}$ for further study.

Multilocus sequence typing (MLST)

To investigate the molecular epidemiology, MLST was performed as described previously by Griffiths et al[18]. The adk, atpA, $d x r, g / y \mathrm{~A}, r e c \mathrm{~A}, \operatorname{sodA}$, and tpi housekeeping genes were targeted. ATCC BAA-1870 
was positive control. The amplified products were sent to Sangon Biotech (Shanghai, China) for sequencing. Then, the DNA sequences of the 7 genes were submitted to the MLST database (http://pubmlst.org/cdifficile) to get the sequence type (ST). The specific primers for toxin genes and housekeeping genes are shown in Table 1. The corresponding PCR characterizations are shown in Fig. 1 and Fig. 2, respectively.

Table 1

Primers used for toxin genes and housekeeping genes of $C$. difficile

\begin{tabular}{|c|c|c|c|}
\hline Gene & Primer & Nucleotide sequence(5'-3') & Fragment length(bp) \\
\hline \multirow[t]{2}{*}{$\operatorname{tcd} A$} & tcdA-F3345 & GCATGATAAGGCAACTTCAGTGGTA & \multirow[t]{2}{*}{625} \\
\hline & tcdA-R3969 & АGTTCCTCСTGCTCCATCAAATG & \\
\hline \multirow[t]{2}{*}{$t c d B$} & tcdB-F5670 & CCAAARTGGAGTGTTACAAACAGGTG & \multirow[t]{2}{*}{410} \\
\hline & tcdB-R6079B & GCATTTCTCCGTTTTCAGCAAAGTA & \\
\hline \multirow[t]{2}{*}{$c d t A$} & cdtA-F739A & GGGAAGCACTATATTAAAGCAGAAGC & \multirow[t]{2}{*}{221} \\
\hline & cdtA-R958 & CTGGGTTAGGATTATTTACTGGACCA & \\
\hline \multirow[t]{2}{*}{$c d t B$} & ctdB-F617 & TTGACCCAAAGTTGATGTCTGATTG & \multirow[t]{2}{*}{262} \\
\hline & ctdB-R878 & CGGATCTCTTGCTTCAGTCTTTATAG & \\
\hline \multirow[t]{2}{*}{$a d k$} & adk1F & TTACTTGGACCTCCAGGTGC & \multirow[t]{2}{*}{501} \\
\hline & adk1R & TTTCCACTTCCTAAGGCTGC & \\
\hline \multirow[t]{2}{*}{ atpA } & atpA1F & TGATGATTTAAGTAAACAAGCTG & \multirow[t]{2}{*}{555} \\
\hline & atpA1R & AATCATGAGTGAAGTCTTCTCC & \\
\hline \multirow[t]{2}{*}{$d x r$} & $d x r 3 F$ & GCTACTTTCCATTCTATCTG & \multirow[t]{2}{*}{411} \\
\hline & $\mathrm{dxr} 4 \mathrm{R}$ & CCAACTCTTTGTGCTATAAA & \\
\hline \multirow[t]{2}{*}{ glyA } & glyA1F & ATAGCTGATGAGGTTGGAGC & \multirow[t]{2}{*}{516} \\
\hline & glyA1R & TTCTAGCCTTAGATTCTTCATC & \\
\hline \multirow[t]{2}{*}{ recA } & recA2F & CAGTAATGAAATTGGGAGAAGC & \multirow[t]{2}{*}{564} \\
\hline & recA2R & ATTCAGCTTGCTTAAATGGTG & \\
\hline \multirow[t]{2}{*}{ sodA } & sodA5F & CCAGTTGTCAATGTATTCATTTC & \multirow[t]{2}{*}{450} \\
\hline & sodA6R & ATAACTTCATTTGCTTTTACACC & \\
\hline \multirow[t]{2}{*}{ tpi } & tpi2F & ATGAGAAAACCTATAATTGCAG & \multirow[t]{2}{*}{504} \\
\hline & tpi2R & TTGAAGGTTTAACACTTCCACC & \\
\hline
\end{tabular}


Clinical data were obtained through the inpatient medical record system, including the length of stay (LOS), submission date, gender, age, diagnosis, stool consistency, history of IBD and so on. Potential risk factors ( 3 months before the detection of $\mathrm{CDI}$ ), including treatment with antibiotics, systemic steroids, oral 5-aminosalicylic acid (5-ASA) and proton pump inhibitor (PPI) were classified as positive or negative, respectively. If IBD patients were complicated with $\mathrm{CDI}$, metronidazole and ornidazole were mostly used to treat CDI. Laboratory test indexes during the hospitalization were also collected.

\section{Statistical analysis}

All data were analyzed using SPSS 22.0 statistical software (SPSS, Chicago, IL, USA). Continuous variables were analyzed by Student's $t$ test or Wilcoxon Rank Sum test, depending on whether data were normally distributed. In this study, age was analyzed by Student's $t$ test and the length of stay was analyzed by Wilcoxon Rank Sum test. Categorical variables were expressed by frequencies and compared through $\chi^{2}$ test. Univariate and multivariate logistic regression were used to identify the independent risk factors for $\mathrm{CDI}$ among patients with IBD. Odds ratios $(\mathrm{OR})$ and $95 \%$ confidence interval $(95 \% \mathrm{Cl})$ were calculated for risk factors to quantify the strength of these associations. Also, $P<0.05$ was considered statistically significant.

\section{Resutlts}

Demographics and Characteristics

A total of 193 cases of IBD patients were confirmed. Patients who did not meet the inclusion criteria were excluded and 134 cases were enrolled in this study finally, including 84 patients with UC and 50 patients with CD. Of these patients, 85 were male and 49 were female. The mean age of IBD patients was 42 years of old and CD patients were much younger than UC patients. The median LOS in CD group was 6 days while the median LOS in UC group was 9 days. There was no statistical significance in terms of gender, antibiotics and PPI between CD patients and UC patients. Also, we found 134 cases of non IBD diarrhea patients as matched group. Although the LOS in IBD group was shorter than that in non IBD group, the incidence of CDI in IBD group was much higher than that in non IBD group and the difference was statistically significant. More details were described in Table 2. 
Table 2

Demographics and characteristics of patients with or without IBD

\begin{tabular}{|c|c|c|c|c|c|c|}
\hline Factors & $\begin{array}{l}\text { IBD } n= \\
134\end{array}$ & $\begin{array}{l}\text { No IBD } n= \\
134\end{array}$ & $P$ & $C D n=50$ & UC $n=84$ & $P$ \\
\hline \multirow{2}{*}{$\begin{array}{l}\text { Age,yrs[Mean } \\
\text { (range)] }\end{array}$} & \multirow{2}{*}{$\begin{array}{l}42.1(16- \\
83)\end{array}$} & 42.4 & \multirow[t]{2}{*}{$0.867^{a}$} & \multirow{2}{*}{$\begin{array}{l}31.7(16- \\
56)\end{array}$} & 48.2 & \multirow{2}{*}{$\begin{array}{l}< \\
0.001^{a *}\end{array}$} \\
\hline & & $(16-83)$ & & & $(19-83)$ & \\
\hline Male [N (\%)] & 85 (63.4\%) & $85(63.4 \%)$ & 1 & $37(74.0 \%)$ & $\begin{array}{l}48 \\
(57.1 \%)\end{array}$ & 0.05 \\
\hline \multirow{2}{*}{$\begin{array}{l}\text { LOS[median } \\
\text { (quartile)] }\end{array}$} & \multirow[t]{2}{*}{$8(5-13.5)$} & \multirow[t]{2}{*}{$10(5-23.25)$} & \multirow[t]{2}{*}{$0.013^{b *}$} & \multirow[t]{2}{*}{$6(5-10.25)$} & & \multirow[t]{2}{*}{$0.009^{b x}$} \\
\hline & & & & & $(6-16)$ & \\
\hline CDI & $33(24.6 \%)$ & $12(9.0 \%)$ & $0.001^{*}$ & $16(32 \%)$ & $17(20.2 \%)$ & 0.126 \\
\hline Antibiotics & $47(35.1 \%)$ & $62(46.3 \%)$ & 0.062 & $17(34 \%)$ & $30(35.7 \%)$ & 0.841 \\
\hline Cephalosporin & $36(26.9 \%)$ & $32(23.9 \%)$ & 0.574 & $12(24 \%)$ & $24(28.6 \%)$ & 0.564 \\
\hline Quinolones & $17(12.7 \%)$ & $10(7.5 \%)$ & 0.155 & $5(10 \%)$ & $12(14.3 \%)$ & 0.471 \\
\hline PPI & $28(20.9 \%)$ & $21(15.7 \%)$ & 0.105 & $6(12 \%)$ & $22(26.2 \%)$ & 0.051 \\
\hline
\end{tabular}

Incidence of CDI in IBD patients

In total, 33 (24.6\%) non-duplicate toxigenic $C$. difficile isolates were identified from 134 IBD patients suffering from diarrhoea while $12(9.0 \%)$ cases of CDI were confirmed among matched non IBD patients. Toxigenic $C$. difficile isolates were both $t c d A$ and $t c d B$ positive and no binary toxin positive strain was isolated in this investigation. There were 4 toxin genes negative strains isolated from UC and CD groups, respectively. More details were described in Fig. 3 and Fig. 4. The incidence of CDI in CD group was 32.0\% $(16 / 50)$ while the incidence of CDI in UC group was $20.2 \%$ (17/84). However, there was no statistically significant about the incidence between $\mathrm{CD}$ and UC group. On the other hand, the incidence of CDI in IBD group was significantly higher than the matched non IBD group. In Fig. 5, the detection rates of CDI in CD and UC group were both higher than the mached groups, respectively.

\section{MLST and source of CDI}

After MLST analysis, 33 toxigenic $C$. difficile isolations from IBD patients could be classified into 9 sequence types (ST). The most prevalent type was ST54 (27.3\%, 9/33), followed by ST3 $(21.2 \%, 7 / 33)$. The hypervirulent $C$. difficile strain ST1 (NAP1/B1/027) was not found in our study. ST54 and ST3 accounted for almost half of the toxigenic $C$. difficile isolations from diarrhea inpatients with IBD in our hospital. In addition, two-thirds of CDI in IBD patients are community-acquired infection (22/33) and nosocomial infections only accounted for 33.3\% (11/33). More details were listed in Table 3 and Fig. 6. 
Table 3

Source of CDI and stool consistency at discharge

\begin{tabular}{|c|c|c|c|c|c|c|c|}
\hline Code & $\begin{array}{l}\text { Admission } \\
\text { time }\end{array}$ & $\begin{array}{l}\text { Isolation } \\
\text { time }\end{array}$ & $\begin{array}{l}\text { Hospital- } \\
\text { acquiredCDI }\end{array}$ & MLST & Type & Antibiotics & $\begin{array}{l}\text { Stool } \\
\text { consistency } \\
\text { at discharge }\end{array}$ \\
\hline CDF1 & 20131006 & 20131023 & Yes & ST35 & UC & No & diarrhea \\
\hline CDF2 & 20131023 & 20131024 & No & ST37 & UC & $L V X+O N Z$ & loose stool \\
\hline CDF3 & 20140120 & 20140122 & No & ST54 & UC & No & normal \\
\hline CDF4 & 20140218 & 20140225 & Yes & ST26 & $C D$ & TNZ & normal \\
\hline CDF5 & 20140625 & 20140626 & No & ST37 & UC & ONZ & normal \\
\hline CDF6 & 20140624 & 20140702 & Yes & ST54 & UC & No & loose stool \\
\hline CDF7 & 20151123 & 20151124 & No & ST35 & $C D$ & No & normal \\
\hline CDF8 & 20160712 & 20160714 & No & ST54 & UC & No & mucous stool \\
\hline CDF9 & 20160714 & 20160715 & No & ST3 & $C D$ & No & loose stool \\
\hline CDF10 & 20160917 & 20160921 & Yes & ST37 & $C D$ & No & normal \\
\hline CDF11 & 20160928 & 20161002 & Yes & ST54 & $\mathrm{CD}$ & No & diarrhea \\
\hline CDF12 & 20161010 & 20161010 & No & ST54 & UC & No & diarrhea \\
\hline CDF13 & 20161116 & 20161118 & No & ST54 & $C D$ & MET & normal \\
\hline CDF14 & 20170209 & 20170210 & No & ST54 & $C D$ & MET & normal \\
\hline CDF15 & 20170403 & 20170404 & No & ST35 & UC & MET & normal \\
\hline CDF16 & 20170403 & 20170404 & No & ST14 & UC & No & normal \\
\hline CDF17 & 20170503 & 20170504 & No & ST2 & UC & No & normal \\
\hline CDF18 & 20170505 & 20170505 & No & ST42 & UC & ONZ & normal \\
\hline CDF19 & 20170519 & 20170523 & Yes & ST2 & $C D$ & No & diarrhea \\
\hline CDF20 & 20170520 & 20170524 & Yes & ST15 & UC & No & normal \\
\hline CDF21 & 20170523 & 20170524 & No & ST35 & UC & MET & normal \\
\hline CDF22 & 20170527 & 20170530 & Yes & ST37 & $\mathrm{CD}$ & MET & normal \\
\hline CDF23 & 20170721 & 20170722 & No & ST3 & UC & No & normal \\
\hline CDF24 & 20170727 & 20170728 & No & ST35 & $C D$ & No & normal \\
\hline CDF25 & 20170905 & 20170906 & No & ST2 & $C D$ & No & normal \\
\hline
\end{tabular}




\begin{tabular}{|llllllll|}
\hline Code & $\begin{array}{l}\text { Admission } \\
\text { time }\end{array}$ & $\begin{array}{l}\text { Isolation } \\
\text { time }\end{array}$ & $\begin{array}{l}\text { Hospital- } \\
\text { acquiredCDI }\end{array}$ & MLST & Type & Antibiotics & $\begin{array}{l}\text { Stool } \\
\text { consistency } \\
\text { at discharge }\end{array}$ \\
\hline CDF26 & 20171005 & 20171011 & Yes & ST54 & CD & No & loose stool \\
\hline CDF27 & 20180105 & 20180106 & No & ST2 & CD & MET & normal \\
\hline CDF28 & 20180319 & 20180320 & No & ST54 & CD & No & diarrhea \\
\hline CDF29 & 20180412 & 20180413 & No & ST3 & CD & No & normal \\
\hline CDF30 & 20180726 & 20180813 & Yes & ST3 & UC & ONZ & normal \\
\hline CDF31 & 20180808 & 20180816 & Yes & ST3 & UC & No & normal \\
\hline CDF32 & 20181126 & 20181127 & No & ST3 & CD & MET & normal \\
\hline CDF33 & 20181228 & 20181228 & No & ST3 & UC & LVX+ ONZ & normal \\
\hline MET metronidazole, TNZ tinidazole, ONZ ornidazole, LVX levofloxacin & & \\
\hline
\end{tabular}

Risk factors of CDI in IBD patients

The effects of CDI associated risk factors, including age, gender, history of IBD, antibiotics, PPI, LOS and so on. The antibiotics uesd in this study were mainly the third generation cephalosporin and levofloxacin. In Table 4, we could found that quinolones and the first year after diagnosis of IBD have statistics significance $(P<0.01)$. Than, after multivariate logistic regression analysis, these variables were confirmed to be the independent risk factors for CDI among diarrha inpatients with IBD. In Table 5, we discoved that patients with IBD who had a history within one year were about 2.7 times more likely to develop CDI than those with a history of more than one year. What's more, IBD patients who recently used quinolones were about 3 times more likely to develop CDI than those who did not. However, PPI, 5-ASA and systemic steroids were not related to $\mathrm{CDI}$ rate. Also, CDI seems to be not associated with older age and longer hospitalization among diarrhea patients with IBD. 
Table 4

Characteristics of IBD patients with or without CDI

\begin{tabular}{|c|c|c|c|}
\hline Factors & $C D I n=33$ & NC $n=101$ & $P$ \\
\hline Male [N (\%)] & $19(57.6 \%)$ & $66(65.3 \%)$ & 0.421 \\
\hline Age,yrs[Mean (range)] & $41.1(17-82)$ & $42.4(16-83)$ & $0.694^{a}$ \\
\hline LOS[median (quartile)] & $6(4-13)$ & $8(5-13.5)$ & $0.143^{b}$ \\
\hline History of IBD within 1 year & $22(66.7 \%)$ & $38(37.6 \%)$ & $0.004^{*}$ \\
\hline \multicolumn{4}{|l|}{ Treatments } \\
\hline Antibiotics & $13(39.4 \%)$ & $34(33.7 \%)$ & 0.549 \\
\hline Cephalosporin & $10(30.3 \%)$ & $26(25.7 \%)$ & 0.608 \\
\hline Quinolones & $9(27.3 \%)$ & $8(7.9 \%)$ & $0.007^{c *}$ \\
\hline 5-ASA & $28(84.8 \%)$ & $79(78.2 \%)$ & 0.410 \\
\hline PPI & $8(24.2 \%)$ & $20(19.8 \%)$ & 0.586 \\
\hline systemic steroids & $6(18.2 \%)$ & $26(25.7 \%)$ & 0.376 \\
\hline \multicolumn{4}{|l|}{ Laboratory test indexes } \\
\hline $\mathrm{CRP}(>8 \mathrm{mg} / \mathrm{ml})$ & $23(69.7 \%)$ & $70(69.3 \%)$ & 0.966 \\
\hline Hypocalcemia & $13(39.4 \%)$ & $44(43.6 \%)$ & 0.674 \\
\hline Anemia & $21(63.6 \%)$ & $63(62.4 \%)$ & 0.897 \\
\hline
\end{tabular}

Table 5

Multivariate Analysis of Risk Factors for CDI among IBD patients

\begin{tabular}{|llll|}
\hline Factors & $\boldsymbol{P}$ & OR & $95 \% \mathrm{Cl}$ \\
\hline History of IBD within 1 year & 0.026 & 2.669 & $1.123-6.344$ \\
\hline Quinolones & 0.048 & 3.048 & $1.011-9.190$ \\
\hline
\end{tabular}

Stool consistency at discharge

In addition to routine treatment for IBD, not all IBD patients complicated with CDI received antiobitic treatment for $\mathrm{CDI}$ in this study. Metronidazole and ornidazole were mostly used to treat CDI. In Table 3, 13 patients received antibiotics targeted $C$. difficile and only 1 patient still had loose stool at discharge. However, 20 patients only treated IBD alone and no antiobitic was taken to treat CDI. When discharged, 
only 11 patients recovered from diarrhea and the rest of them still had some issues, for example, 1 patient had mucous stool, 3 patients had loose stool, and 5 patients still had diarrhea.

\section{Discussion}

CDI has become a major public health problem. In United States, CDI causes about 450,000 infections and 35,000 deaths each year and $C$. difficile is also the major cause of hospital acquired infections in UK $[19,20]$. Aging population and widely use of antibiotics are the current social situation in China, which can increase the risk of CDI. According to one meta-analysis by our early work, the incidence of CDI among hospitalized diarrhea patients is about $14 \%$ in mainland China[4]. Among IBD patients, the infection rate of $C$. difficile has been increasing steadily over the past decades[8,21]. IBD is proven to be an independent risk factor for CDI. Moreover, IBD populations complicated with CDI usually have worse outcome[22]. In this study, the incidence of CDI among IBD patients was $24.6 \%$ (33/134) and was much higher than matched non IBD group. According to our knowldege, the infection rate of $C$. difficile in patients with IBD most studies reported in western countries is less than $10 \%$ and was much lower than this investigation[23,24]. The method they used to detect CDI was toxigenic culture or an algorithm, including glutamate dehydrogenase $(\mathrm{GDH})$ and $C$. difficile toxin $\mathrm{A}$ or $\mathrm{B}(\mathrm{CDAB})$ with nucleic acid amplification testing (NAAT) as confirmatory test, respectively. The method we used in this study was also toxigenic culture. The rates of CDI between our study and thier researches are comparable. However, while camparing the incidence of our study with other data in China, only few studies about the epidemiology of CDI among IBD patiens in China were searched. One study from Peking Union Medical College Hospital reported that thay identified 60 (7.41\%) cases of CDI among 810 patients with IBD[25]. However, the method they used to detect CDI is enzyme immunoassay (EIA)-based stool test results for CDAB. It is widely reported that the low sensitivity of this method detecting free toxin in stool ranges from $32 \%-79 \%[26-28]$. This may leads to the incidence of $C D I$ on the low side. In our study, we also used VIDAS ${ }^{\circledR}$ C. difficile panel (BioMérieux, France) to detect partial diarrhea samples. Among 29 cases of toxigenic $C$. difficile diarrhea samples detected by toxigenic culture, only 6 cases were positive and 2 cases were suspected for $\mathrm{CDAB}$ from diarrhea samples detected by EIA method. The rest test reselts were all negative. The sensitivity of CDAB for CDI detection compared with toxigenic culture is only $27.6 \%$ (8/29), even including the suspicious positive cases. Although the number of CDI cases included in this study for CDAB test was small, it could also reflect the issue to some extent. So, the real incidence of CDI in hspitalized diahhrea patients with IBD may be higher in China and our data could fill the gap in this field to a certain extent. In this study, about two thirds of CDI among IBD patients were community acquired infections and nosocomial infections only accounted for $33.3 \%(11 / 33)$. According to the research of Rodemann JF and his colleagues, nearly two-thirds of IBD patients with CDI were community acquired infection, which was consistent with our result[21]. Community acquired infection accounts for the majority of CDI among diarrhea inpatients with IBD.

In addition, no binary toxin positive strains were isolated in this study and all toxigenic strains were both positive for $t c d A$ and $t c d B$ and there was no report about the hypervirulent strain RT027 isolated from IBD patients in China as far as we know. ST54 was the main type of toxigenic $C$. difficile among diarrhea 
patients with IBD. After MLST analysis, we discovered that ST54 and ST3 accounted for nearly half of the toxigenic isolations from patients with IBD in this study. Meanwhile, many literatures reported that ST54 was widely distributed in the world and was detected in Japan, India, Chile and other places[29]. It has also been reported that ST54 occupies the second place of CDI in domestic pregnant women[30]. Recently, investigations carried out in Chinese hospitals revealed that ST54 was the predominant type of $\mathrm{CDI}$ in diarrhea patients[31,32]. Also, according to our previous meta-analysis about the domestic molecular epidemiology, the epidemic strain of $C$. difficile isolated from diarrhea patients were mainly ST3, ST37 and ST54, accounting for $18.1 \%, 17.2 \%$ and $16.7 \%$, respectively. This indicates that ST54 strain plays an important role not only in IBD patients, but also in other populations.

It is widely acknowledeged that broad-spectrum antibiotics, proton pump inhibitors, older age, immunosuppressants and long-term hospitalization are all related to the occurrence and development of CDI[33-35]. However, the incidence of CDI among patients with IBD is still very high even if they have not used antibiotics recently and have not been exposed to the medical environment[36]. Compared with the general population, IBD seems to be an independent risk factor for CDI. This may be caused by the imbalance of gastrointestinal and immune functions, intestinal flora disorders, intestinal epithelial damage and other factors in IBD patients. The differences and associations about the risk factors for CDI between IBD patients and the general population deserve further study. In this study, the independent risk factors for CDI among IBD patients were the recent use of quinolones and the first year after initial diagnosis of IBD. In this study, levofloxacin was the mainly uesd quinolones. Quinolones have been widely recognized as an independent risk factor for $\mathrm{CDI}$ and our study confirmed that quinolones remaind a risk factor for $\mathrm{CDI}$ among diarrhea patients with IBD. Singh $\mathrm{H}$ and his colleagues reported the highest rates of $\mathrm{CDI}$ within the first year of IBD diagnosis and shorter duration of IBD was associated with an increased risk of CDI[13]. This may be explained by the higher rates of dysbiosis of gut flora at IBD diagnosis, untreated altered humoral immune responses, or epithelial dysfunction predisposing to CDI. Interestingly, many studies about the independent risk factors for CDI in IBD patients discovered that steroids, biologics and immunomodulators, which have been widely considered as risk factors for CDI, have not been associated with the higher incidence of CDI in IBD patients[24, 37, 38]. In our study, systemic steroids were not associated with CDI susceptibility in patients with IBD. There is no consensus on the independent risk factors for CDI among IBD patients at present. Different studies often come to different conclusions, such as recent use of antibiotics, admission to hospital, PPI and so on[23, 24, 37, 39].

Also, this study did not find that IBD patients complicated with CDI extended the length of hospital stay, which was consistent with the results of multiple single-center studies[8, 40]. One investigation carried out in USA discovered that more than half of the infected IBD patients required hospitalization and $20 \%$ required colectomy. However, only several cases in this study accepted colectomy. Two studies from Europe reported the rate of colectomy was about $5 \%$. There is still controversy over whether CDI increases the rate of colectomy among patients with IBD. High quality researches are needed to resolve this issue. 
What's more, almost all infected IBD patients who received CDI treatment were recovered from diarrhea in this study (12/13) while almost half of the untreated CDI patient still had issues with stool $(9 / 20)$, even 5 cases still had diarrhea when they were at discharge. This indicates the conventional therapy of IBD does not apply to patients complicated with $\mathrm{CDI}$ and emphasizes the importance of early diagnosis and targeted treatment against CDI.

Despite the discoveries in this study, there are some limitations should be considered. Firstly, this singlecenter study was conducted in a tertiary care university teaching hospital and the sample size enrolled in this study was insufficiently large. For example, when analysis the clinical characteristics of IBD patients with or without CDI, cases of some variables are limited. In-depth subgroup analysis about the incidence of CDI among different characteristics of IBD could not be performed. Secondly, not all diarrhea amples used $C D A B$ to detect $C D I$ because this panel was used in our hospital not from the beginning of this study and we could not compare the incidence of CDI with the data from the Beijing, China[25]. Addiotionally, not all diarrhea inpatients sent stool for CDI detection within $48 \mathrm{~h}$ after admission and the true proportion of community-acquired CDI maybe higher than the data we received. Last but not least, we did not follow up the enrolled patients because part patients were from other cities and lost to follow up.

\section{Conclusions}

Although no hypervirulent strain RT027 was detected in this study, the incidence of CDI in IBD patients in our hospital was significantly higher than that in most western countries. Community acquired infection was the main type of CDI and ST54 was the epidemic strains among IBD patients in China. In addition, if the duration of IBD is within one year or quinolone antibiotics have been used recently, clinicians should consider the possibility of IBD patients complicated by CDI and adjust the treatment plan.

\section{Abbreviations}

CDI

Clostridioides difficile infection

IBD

inflammatory bowel disease

MRSA

methicillin-resistant Staphylococcus aureus

UC

ulcerative colitis

CD

Crohn's disease

MLST

multilocus sequence typing

ST

sequence type 
LOS

length of stay

5-ASA

5-aminosalicylic acid

PPI

proton pump inhibitor

OR

odds ratios

$\mathrm{GDH}$

glutamate dehydrogenase

CDAB

C.difficile toxin $\mathrm{A}$ or $\mathrm{B}$

NAAT

nucleic acid amplification testing

\section{Declarations}

\section{Availability of data and materials}

All data generated and analyzed during this study were included in this published article.

Ethics approval and consent to participate

This study was approved by the hospital ethics committee.

Consent for publication

Not applicable.

Competing interests

The authors declare that they have no competing interests.

\section{Funding}

This research received no specific grant from any funding agency in the public, commercial, or not-forprofit sectors.

\section{Author Contributions}


Genyan Liu and Chenjie Tang devised the study. Chengcheng Liu, Yaping Han and Chenjie Tang collected the specimens and/or performed the experiments. Xiaohui Zhang and Wenying Xia collected and analysed data. Chenjie Tang, Fang $\mathrm{Ni}$ and Genyan Liu drafted the manuscript. All authors contributed to and approved the final version.

\section{Acknowledgments}

Not applicable

\section{References}

1. Bartlett JG, Chang TW, Gurwith M, Gorbach SL, Onderdonk AB. Antibiotic-associated pseudomembranous colitis due to toxin-producing clostridia. N Engl J Med. 1978;298(10):531-4.

2. Ricciardi R, Rothenberger DA, Madoff RD, Baxter NN. (2007) Increasing prevalence and severity of Clostridium difficile colitis in hospitalized patients in the United States. Archives of surgery (Chicago, III: 1960) 142 (7):624-631; discussion 631.

3. Asha NJ, Tompkins D, Wilcox MH. Comparative analysis of prevalence, risk factors, and molecular epidemiology of antibiotic-associated diarrhea due to Clostridium difficile, Clostridium perfringens, and Staphylococcus aureus. J Clin Microbiol. 2006;44(8):2785-91.

4. Tang $C$, Cui L, Xu Y, Xie L, Sun P, Liu C, Xia W, Liu G. The incidence and drug resistance of Clostridium difficile infection in Mainland China: a systematic review and meta-analysis. Scientific reports. 2016;6:37865.

5. Wang P, Zhou Y, Wang Z, Xie S, Zhang T, Lin M, Li R, Tan J, Chen Y, Jiang B. Identification of Clostridium difficile ribotype 027 for the first time in Mainland China. Infect Control Hosp Epidemiol. 2014;35(1):95-8.

6. Cheng JW, Xiao M, Kudinha T, Xu ZP, Hou X, Sun LY, Zhang L, Fan X, Kong F, Xu YC. The First Two Clostridium difficile Ribotype 027/ST1 Isolates Identified in Beijing, China-an Emerging Problem or a Neglected. Threat? Scientific reports. 2016;6:18834.

7. Tamboli CP, Neut C, Desreumaux P, Colombel JF. Dysbiosis as a prerequisite for IBD. Gut. 2004;53(7):1057.

8. Issa M, Vijayapal A, Graham MB, Beaulieu DB, Otterson MF, Lundeen S, Skaros S, Weber LR, Komorowski RA, Knox JF, Emmons J, Bajaj JS, Binion DG. Impact of Clostridium difficile on inflammatory bowel disease. Clinical gastroenterology hepatology: the official clinical practice journal of the American Gastroenterological Association. 2007;5(3):345-51.

9. Nguyen GC, Kaplan GG, Harris ML, Brant SR. A national survey of the prevalence and impact of Clostridium difficile infection among hospitalized inflammatory bowel disease patients. Am J Gastroenterol. 2008;103(6):1443-50. 
10. Powell N, Jung SE, Krishnan B. Clostridium difficile infection and inflammatory bowel disease: a marker for disease extent? Gut. 2008;57(8):1183-4. author reply 1184.

11. Ananthakrishnan AN, Issa M, Binion DG. Clostridium difficile and inflammatory bowel disease. Gastroenterol Clin N Am. 2009;38(4):711-28.

12. Ananthakrishnan AN, McGinley EL, Saeian K, Binion DG. Temporal trends in disease outcomes related to Clostridium difficile infection in patients with inflammatory bowel disease. Inflamm Bowel Dis. 2011;17(4):976-83.

13. Singh H, Nugent Z, Yu BN, Lix LM, Targownik LE, Bernstein CN. Higher Incidence of Clostridium difficile Infection Among Individuals With Inflammatory Bowel Disease. Gastroenterology. 2017;153(2):430-8. e432.

14. McDonald LC, Gerding DN, Johnson S, Bakken JS, Carroll KC, Coffin SE, Dubberke ER, Garey KW, Gould CV, Kelly C, Loo V, Shaklee Sammons J, Sandora TJ, Wilcox MH. Clinical Practice Guidelines for Clostridium difficile Infection in Adults and Children: 2017 Update by the Infectious Diseases Society of America (IDSA) and Society for Healthcare Epidemiology of America (SHEA). Clinical infectious diseases: an official publication of the Infectious Diseases Society of America. 2018;66(7):987-94.

15. Surawicz CM, Brandt LJ, Binion DG, Ananthakrishnan AN, Curry SR, Gilligan PH, McFarland LV, Mellow M, Zuckerbraun BS. Guidelines for Diagnosis, Treatment and Prevention of Clostridium difficile Infections. Am J Gastroenterol. 2013;108(4):478-98.

16. Tang C, Li Y, Liu C, Sun P, Huang X, Xia W, Qian H, Cui L, Liu G. Epidemiology and risk factors for Clostridium difficile-associated diarrhea in adult inpatients in a university hospital in China. Am J Infect Control. 2018;46(3):285-90.

17. Persson S, Torpdahl M, Olsen KE. New multiplex PCR method for the detection of Clostridium difficile toxin $A(\operatorname{tcd} A)$ and toxin $B(t c d B)$ and the binary toxin (cdtA/cdtB) genes applied to a Danish strain collection. Clinical microbiology infection: the official publication of the European Society of Clinical Microbiology Infectious Diseases. 2008;14(11):1057-64.

18. David G, Warren F, Melina K, Rory B, Crook DW, Rowena F, Tanya G, Harding RM, Jeffery KJM, Jolley KA. Multilocus sequence typing of Clostridium difficile. J Clin Microbiol. 2010;48(3):770.

19. Lessa FC, Mu Y, Bamberg WM, Beldavs ZG, Dumyati GK, Dunn JR, Farley MM, Holzbauer SM, Meek JI, Phipps EC, Wilson LE, Winston LG, Cohen JA, Limbago BM, Fridkin SK, Gerding DN, McDonald LC. Burden of Clostridium difficile infection in the United States. N Engl J Med. 2015;372(9):825-34.

20. Bartlett JG, Moon N, Chang TW, Taylor N, Onderdonk AB. Role of Clostridium difficile in antibioticassociated pseudomembranous colitis. Gastroenterology. 1978;75(5):778-82.

21. Rodemann JF, Dubberke ER, Reske KA, Seo DH, Stone CD. Incidence of Clostridium difficile infection in inflammatory bowel disease. Clinical gastroenterology hepatology: the official clinical practice journal of the American Gastroenterological Association. 2007;5(3):339-44.

22. Zhang $T$, Lin QY, Fei JX, Zhang Y, Lin MY, Jiang SH, Wang P, Chen Y. Clostridium Difficile Infection Worsen Outcome of Hospitalized Patients with Inflammatory Bowel Disease. Scientific reports. 
2016;6:29791.

23. Regnault H, Bourrier A, Lalande V, Nion-Larmurier I, Sokol H, Seksik P, Barbut F, Cosnes J, Beaugerie L. (2014) Prevalence and risk factors of Clostridium difficile infection in patients hospitalized for flare of inflammatory bowel disease: a retrospective assessment. Digestive and liver disease: official journal of the Italian Society of Gastroenterology and the Italian Association for the Study of the Liver 46 (12):1086-1092.

24. Maharshak N, Barzilay I, Zinger H, Hod K, Dotan I. Clostridium difficile infection in hospitalized patients with inflammatory bowel disease: Prevalence, risk factors, and prognosis. Medicine. 2018;97(5):e9772.

25. Li Y, Xu H, Xu T, Xiao M, Tang H, Wu D, Tan B, Li J, Yang H, Lv H, Xu Y, Qian J. Case-Control Study of Inflammatory Bowel Disease Patients with and without Clostridium difficile Infection and Poor Outcomes in Patients Coinfected with C. difficile and Cytomegalovirus. Digestive diseases sciences. 2018;63(11):3074-83.

26. Crobach MJ, Dekkers OM, Wilcox MH, Kuijper EJ. (2009) European Society of Clinical Microbiology and Infectious Diseases (ESCMID): data review and recommendations for diagnosing Clostridium difficile-infection (CDI). Clinical microbiology and infection: the official publication of the European Society of Clinical Microbiology and Infectious Diseases 15 (12):1053-1066.

27. Planche T, Aghaizu A, Holliman R, Riley P, Poloniecki J, Breathnach A, Krishna S. Diagnosis of Clostridium difficile infection by toxin detection kits: a systematic review. The Lancet Infectious diseases. 2008;8(12):777-84.

28. Eastwood K, Else P, Charlett A, Wilcox M. Comparison of nine commercially available Clostridium difficile toxin detection assays, a real-time PCR assay for $\mathrm{C}$. difficile tcdB, and a glutamate dehydrogenase detection assay to cytotoxin testing and cytotoxigenic culture methods. $\mathrm{J}$ Clin Microbiol. 2009;47(10):3211-7.

29. Plaza-Garrido A, Barra-Carrasco J, Macias JH, Carman R, Fawley WN, Wilcox MH, Hernandez-Rocha C, Guzman-Duran AM, Alvarez-Lobos M, Paredes-Sabja D. Predominance of Clostridium difficile ribotypes 012, 027 and 046 in a university hospital in Chile, 2012. Epidemiol Infect. 2016;144(5):976-9.

30. Ye GY, Li N, Chen YB, Lv T, Shen P, Gu SL, Fang YH, Li LJ. Clostridium difficile carriage in healthy pregnant women in China. Anaerobe. 2016;37:54-7.

31. Zhou Y, Mao L, Yu J, Lin Q, Luo Y, Zhu X, Sun Z. Epidemiology of Clostridium difficile infection in hospitalized adults and the first isolation of C. difficile PCR ribotype 027 in central China. BMC Infect Dis. 2019;19(1):232.

32. Alvarez-Perez S, Harmanus C, Kuijper EJ, Garcia ME, Chen YB, Gu SL, Shen P, Lv T, Fang YH, Tang LL, Li LJ. Molecular epidemiology and antimicrobial susceptibility of Clostridium difficile isolated from hospitals during a 4-year period in China. Zoonoses public health. 2018;67(1):52-9.

33. Moshkowitz M, Ben-Baruch E, Kline Z, Shimoni Z, Niven M, Konikoff F. Risk factors for severity and relapse of pseudomembranous colitis in an elderly population. Colorectal disease: the official journal 
of the Association of Coloproctology of Great Britain Ireland. 2007;9(2):173-7.

34. Dial S, Delaney JA, Schneider V, Suissa S. (2006) Proton pump inhibitor use and risk of communityacquired Clostridium difficile-associated disease defined by prescription for oral vancomycin therapy. CMAJ: Canadian Medical Association journal = journal de l'Association medicale canadienne 175 (7):745-748.

35. Dial S, Kezouh A, Dascal A, Barkun A, Suissa S. (2008) Patterns of antibiotic use and risk of hospital admission because of Clostridium difficile infection. CMAJ: Canadian Medical Association journal = journal de l'Association medicale canadienne 179 (8):767-772.

36. Epple HJ. Therapy- and non-therapy-dependent infectious complications in inflammatory bowel disease. Digestive diseases (Basel Switzerland). 2009;27(4):555-9.

37. Sokol H, Lalande V, Landman C, Bourrier A, Nion-Larmurier I, Rajca S, Kirchgesner J, Seksik P, Cosnes J, Barbut F, Beaugerie L. (2017) Clostridium difficile infection in acute flares of inflammatory bowel disease: A prospective study. Digestive and liver disease: official journal of the Italian Society of Gastroenterology and the Italian Association for the Study of the Liver 49 (6):643-646.

38. Micic D, Yarur A, Gonsalves A, Rao VL, Broadaway S, Cohen R, Dalal S, Gaetano JN, Glick LR, Hirsch A, Pekow J, Sakuraba A, Walk ST, Rubin DT. Correction to: Risk Factors for Clostridium difficile Isolation in Inflammatory Bowel Disease: A Prospective Study. Digestive diseases sciences. 2018;63(10):2815.

39. Micic D, Yarur A, Gonsalves A, Rao VL, Broadaway S, Cohen R, Dalal S, Gaetano JN, Glick LR, Hirsch A, Pekow J, Sakuraba A, Walk ST, Rubin DT. Risk Factors for Clostridium difficile Isolation in Inflammatory Bowel Disease: A Prospective Study. Digestive diseases sciences. 2018;63(4):101624.

40. Jodorkovsky D, Young Y, Abreu MT. Clinical outcomes of patients with ulcerative colitis and coexisting Clostridium difficile infection. Digestive diseases sciences. 2010;55(2):415-20.

\section{Figures}


A

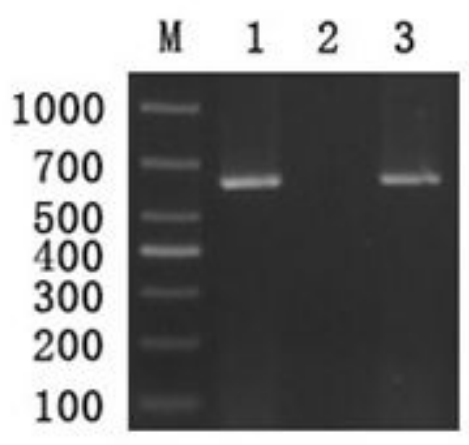

C

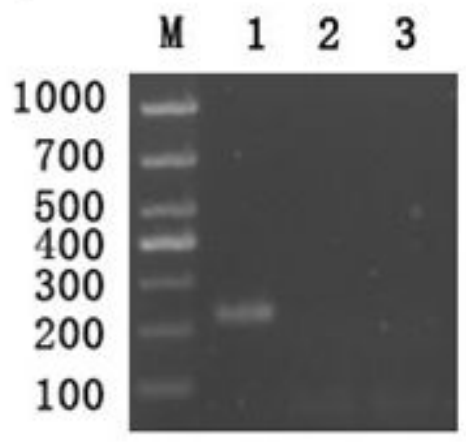

B

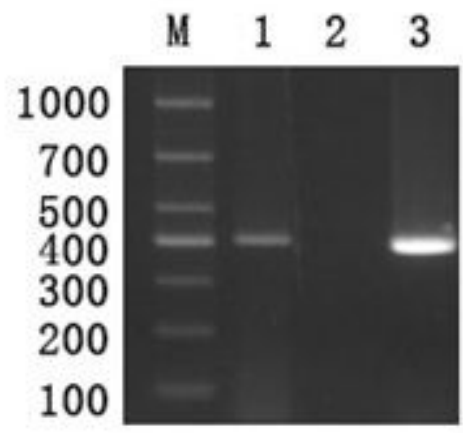

D

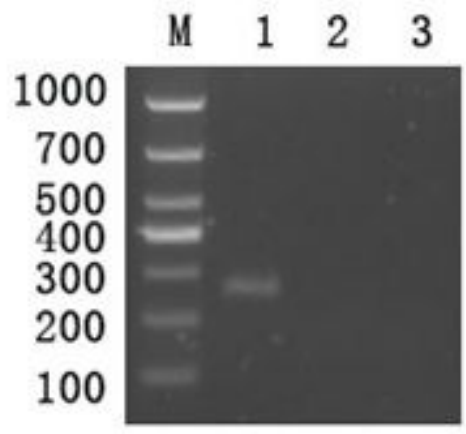

\section{Figure 1}

PCR products of C. difficile toxin genes. (A) PCR products of tcdA (625bp), (B) PCR products of tcdB (410bp), (C) PCR products of cdtA (221bp), (D) PCR products of cdtB (262bp), M molecular marker, 1 positive control, 2 negative control, 3 clinical samples

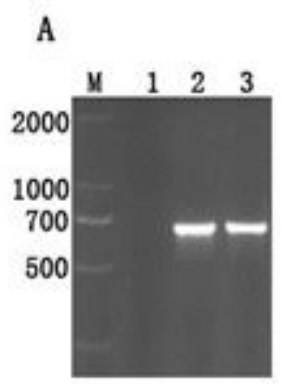

B

C

D
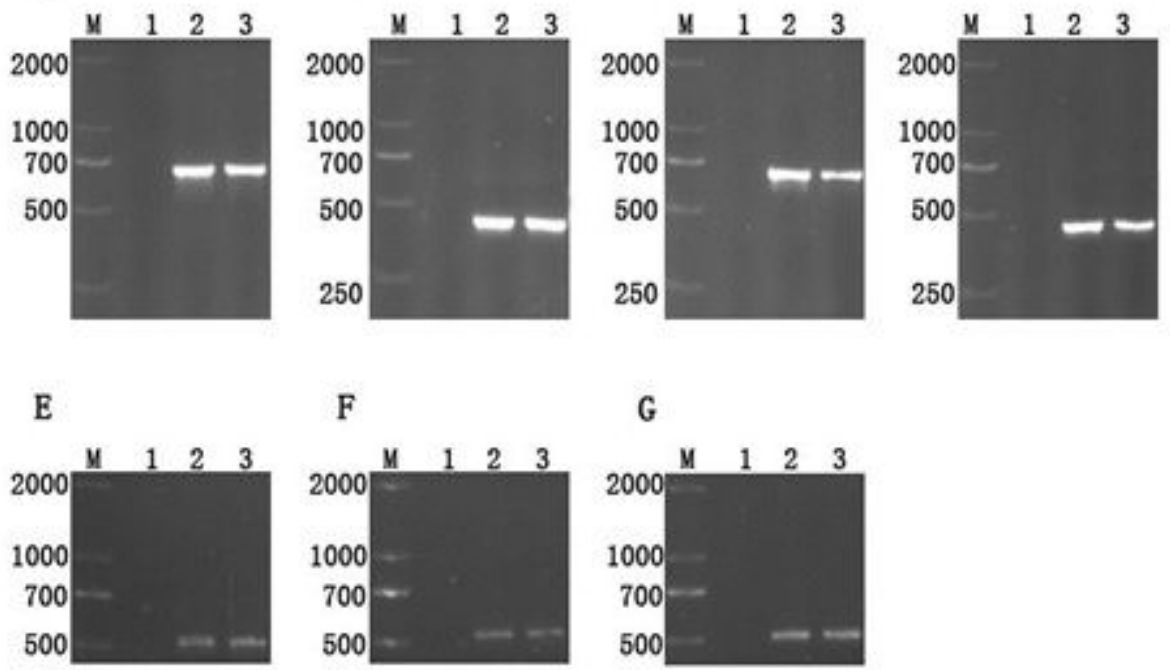

F

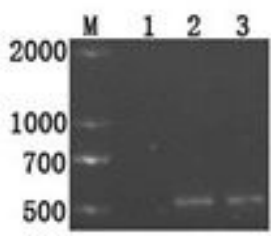

G

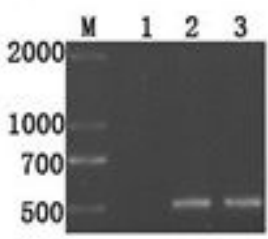

Figure 2 
PCR products of C. difficile MLST. (A) PCR products of atpA (555bp), (B) PCR products of dxr (411bp), (C) PCR products of recA (564bp), (D) PCR products of sodA(450bp), (E) PCR products of adk (501bp), (F) PCR products of glyA(516bp), (G) PCR products of tpi (504bp), M molecular marker, 1 negative control, 2 positive control, 3 clinical samples

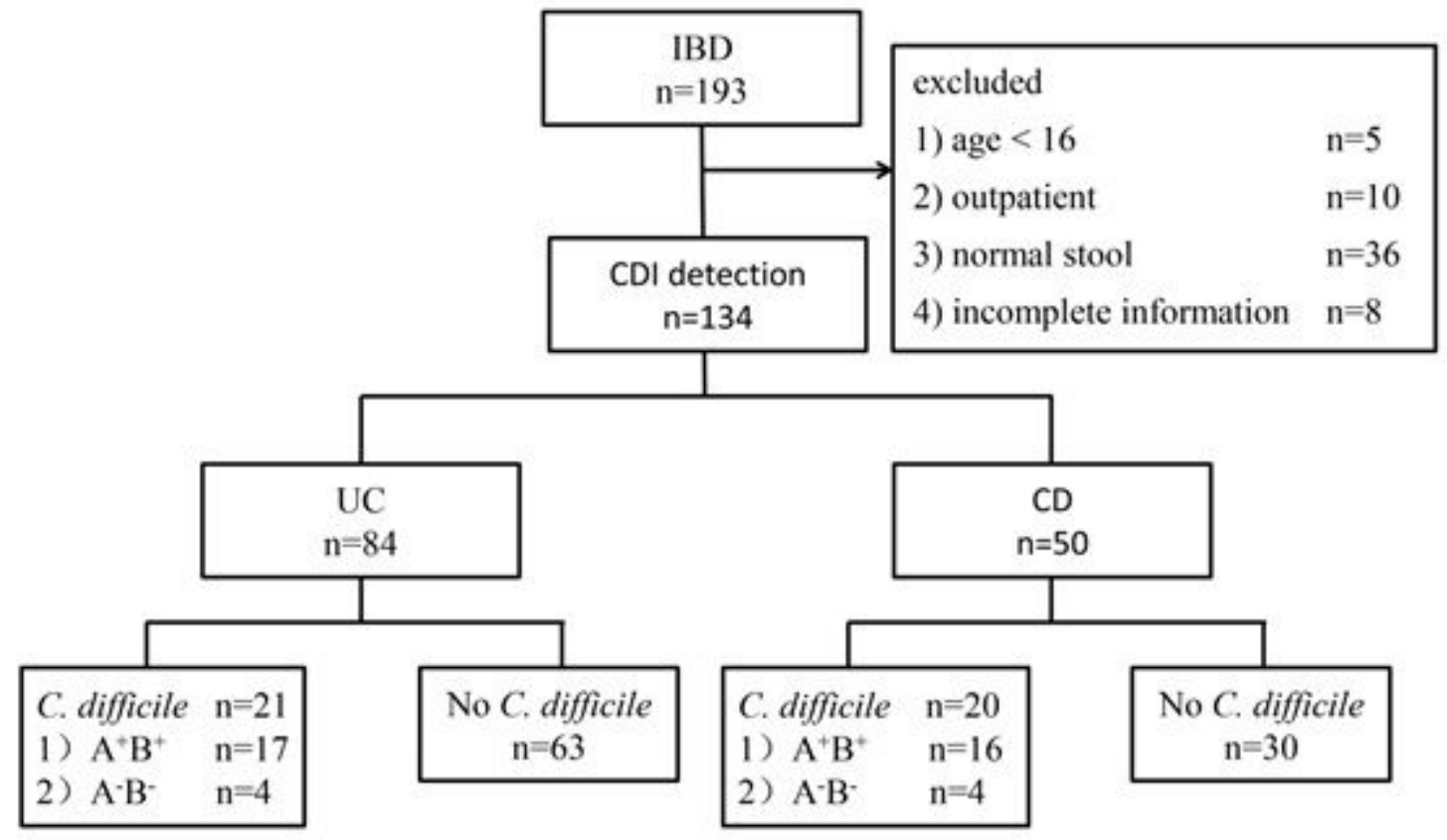

Figure 3

Overview diagram of the detcetion of $C D I$ in IBD patients. $A+B+$ positive results of tcdA and tcdB, A-Bnegative reslults of $t c d A$ and $t c d B$

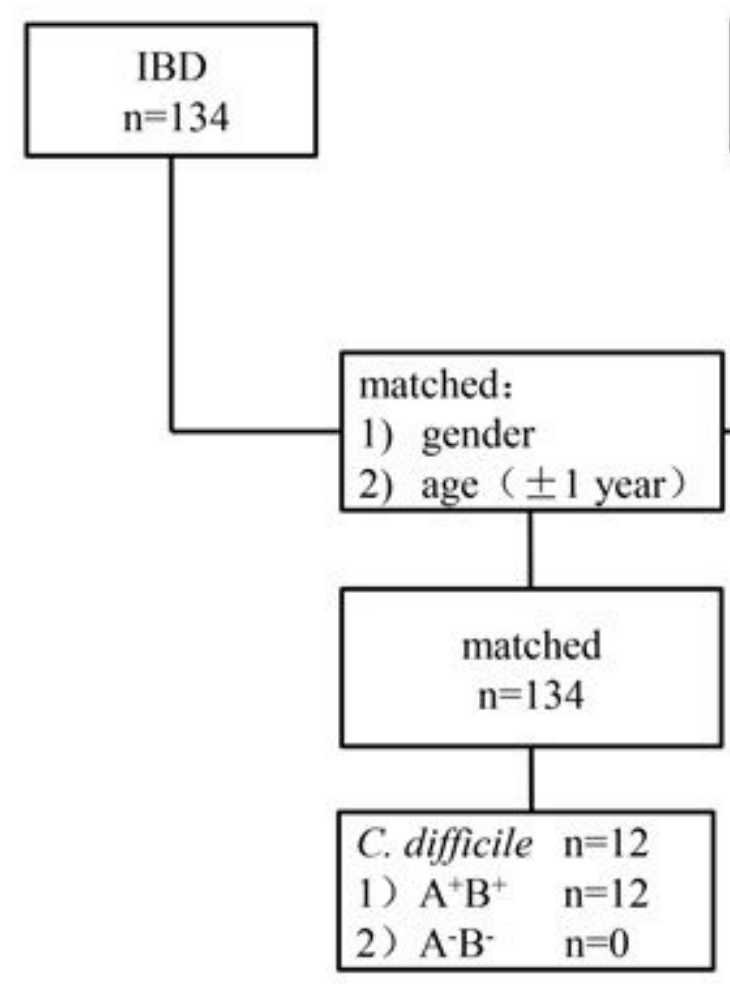


Figure 4

Overview diagram of the detcetion of $C D I$ in non IBD patients. $A+B+$ positive results of tcdA and tcdB, A-Bnegative reslults of $t c d A$ and $t c d B$

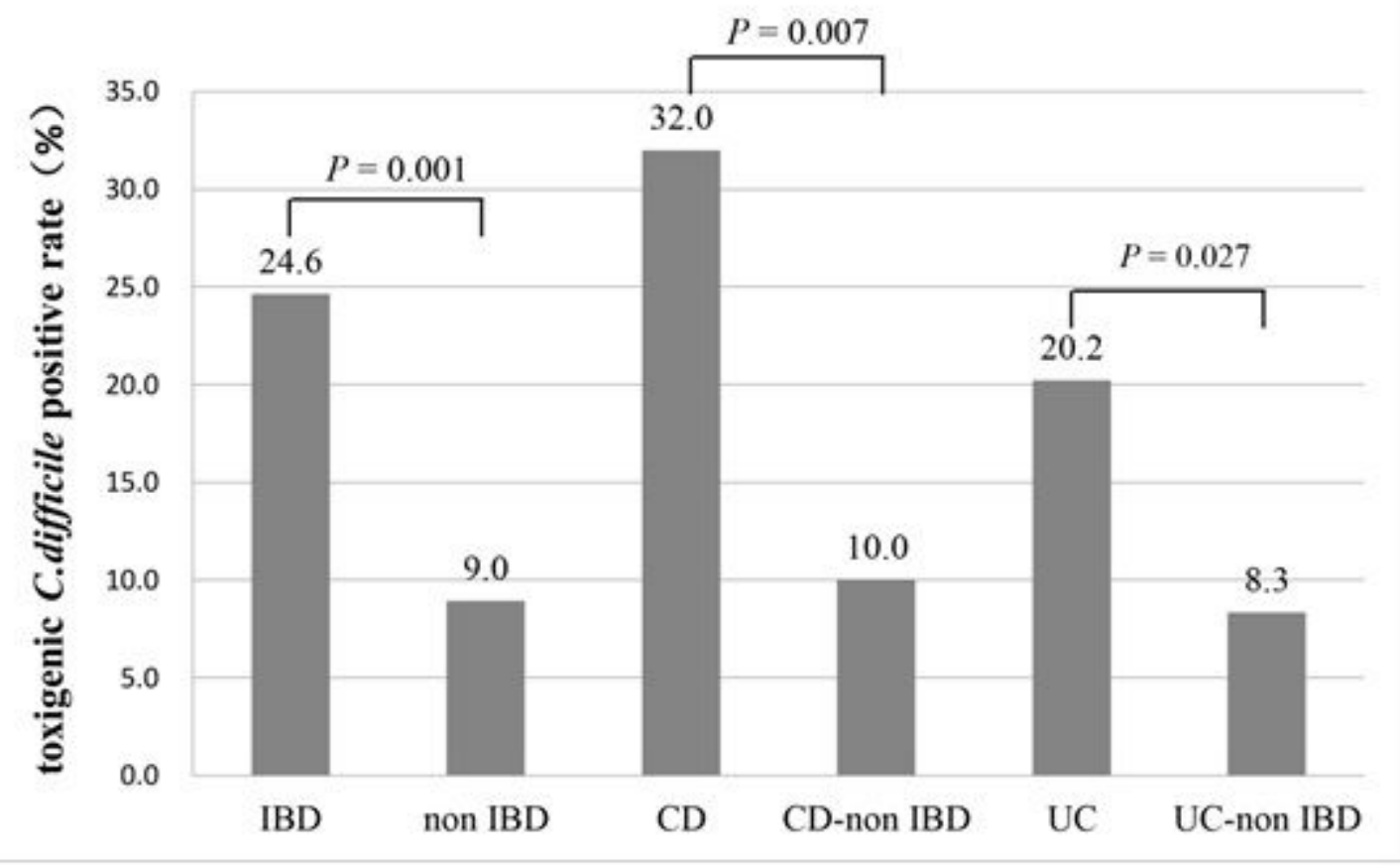

Figure 5

Detection of CDI in IBD patients and matched non IBD patients 
A

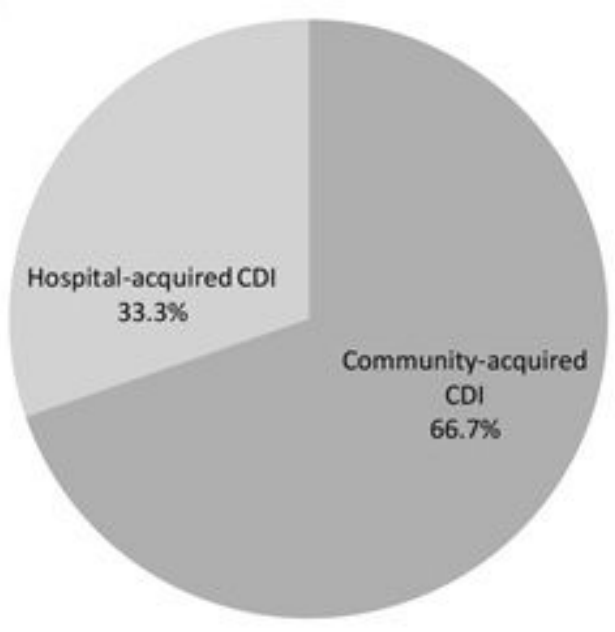

$\mathrm{C}$

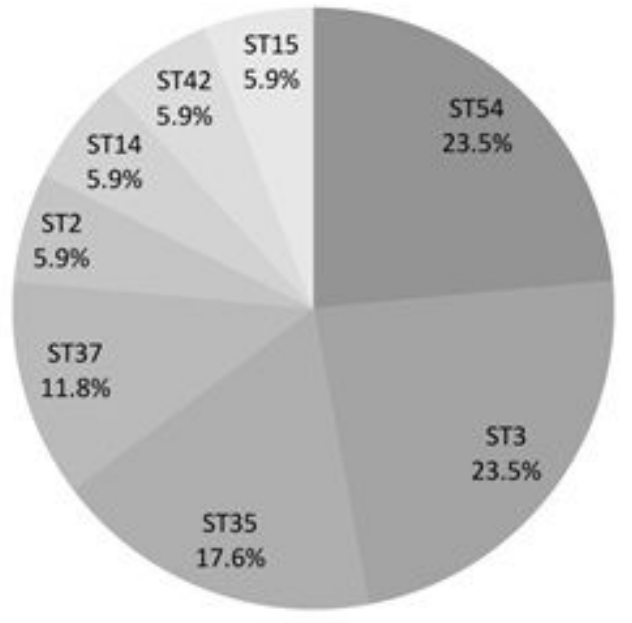

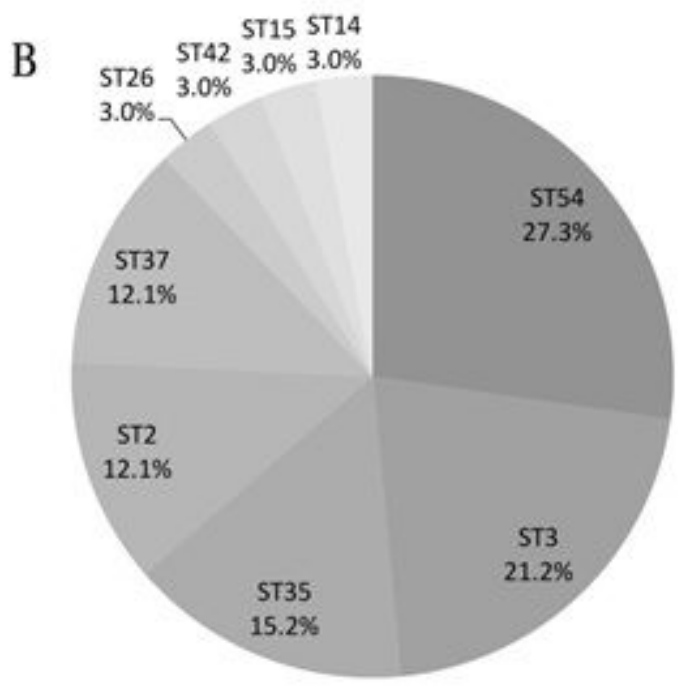

D

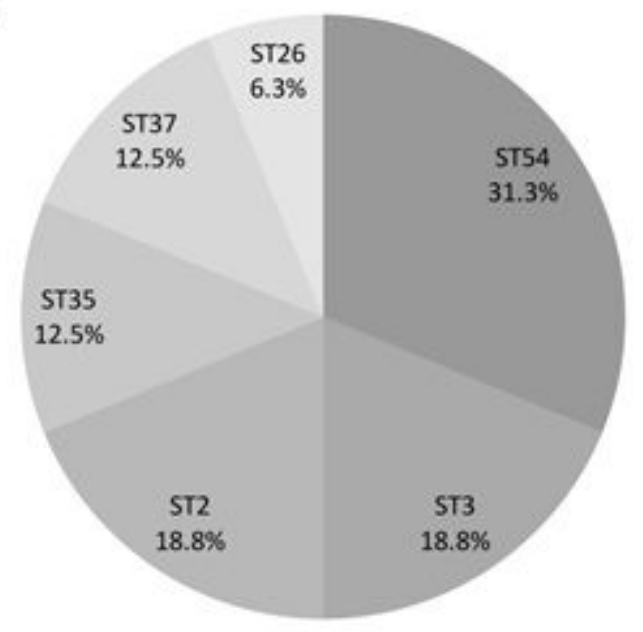

Figure 6

Source and MLST analysis of CDI. A the source of CDI $n=33, B$ MLST of IBD patients $n=33, C$ MLSTof UC patients $n=17, D$ MLST of $C D$ patients $n=16$ 